\title{
Research Article Enhanced Gain Microstrip Patches Operating on Higher Order Modes
}

\author{
Milan Polivka and Alois Holub \\ Received 4 May 2007; Accepted 10 July 2007 \\ Recommended by Kamya Yekeh Yazdandoost \\ Novel topologies of rectangular microstrip patches providing broadside radiated one point fed radiators with enhanced gain are \\ compared. The principle of the gain enhancement is based on an extension of a source area. Dominant in-phase current distribu- \\ tion on the patch is maintained by using higher order mode and geometrical modification of the patch topology. That is achieved \\ by introducing suitable perturbation elements in the shape of slots and notches. Two principal patch topologies operating on $\mathrm{TM}_{21}$ \\ and $\mathrm{TM}_{03}$ modes are described. A comparison of simulated and measured properties of realized prototypes at $10 \mathrm{GHz}$ band are \\ presented. The results show that gain $12.3 \mathrm{dBi}$ of single patch can be reached.
}

Copyright (c) 2007 M. Polivka and A. Holub. This is an open access article distributed under the Creative Commons Attribution License, which permits unrestricted use, distribution, and reproduction in any medium, provided the original work is properly cited.

\section{INTRODUCTION}

In various communication and measurement applications, there is a need of planar radiators with enhanced gain. Microstrip patch radiators exhibit a gain of an approximate range of 5-10 dBi [1], depending on several parameters such as the properties of the used substrate (height, relative permittivity), the width/length ratio $(W / L)$ of the patch radiator, and the dimensions of the ground plane. There are several standard techniques to enhance the gain, including the use of superstrate dielectric layers with high permittivity $[2,3]$, stacked parasitic patch arrangement [4], or extension of effective aperture by localization of higher-order modes in fractal patch antennas $[5,6]$. The novel technique presented here has similar physical basis to that reported in $[5,6]$. It is based on extension of a source area, maintaining in-phase current distribution of higher-order mode on the dominant part of the patch. The presented concept uses perturbation elements (PE) in the shape of slots and notches in the patch topology for the modification of surface current density of the operational mode. Due to the existence of two or more in-phase source areas, these radiators actually behave similar to an antenna array while maintaining one feeding point.

Here we present two principal patch topologies for a gain enhancement of rectangular patch. The first patch topology is modified by a pair of perturbation elements in the shape of T-notch [7] and operates on $\mathrm{TM}_{21}$ mode. The second topology is inspired by Franklin's idea of collinear wire array [8]. It employs patch with $\mathrm{TM}_{03}$ mode in a collinear [9] or planar collinear arrangement $[10,11]$. The principle of the operation is explained via description of vector surface current distribution of individual patches. A comparison of simulated and measured properties of realized prototypes designed at $10 \mathrm{GHz}$ band is presented.

\section{ANTENNA DESIGN AND PRINCIPLE OF OPERATION}

\subsection{Description of the antenna prototypes}

Three enhanced gain patch topologies have been designed at $10 \mathrm{GHz}$ to demonstrate their gain improvement compared to a reference rectangular patch (see Figure 1): T-notched patch operating on $\mathrm{TM}_{21}$ mode, collinear microstrip patch antenna, and its planar extension (CoMPA and PCoMPA) operating on $\mathrm{TM}_{03}$ mode at $10 \mathrm{GHz}$. Their geometry can be seen in Figures 1(b)-1(d).

Patch radiators have been realized from metal sheet with thickness $t=0.3 \mathrm{~mm}$ placed in height $h=2.7 \mathrm{~mm}$ (approx. $0.09 \lambda$ ) over the ground plane (see Figure 2). To maintain the radiator construction as simple as possible, any capacitive compensation of probe inductance has not been performed. SMA connector has been attached to the bottom of the ground plane with the size of $40 \times 40 \mathrm{~mm}$ for reference and T-notched patches and with the size of $50 \times 50 \mathrm{~mm}$ for CoMPA and PCoMPA patches. 


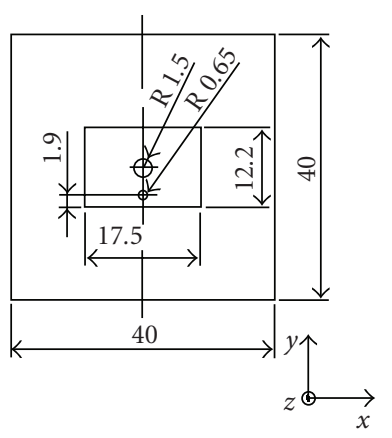

(a)

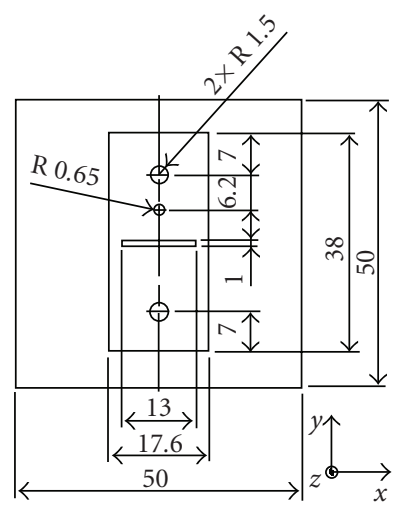

(c)

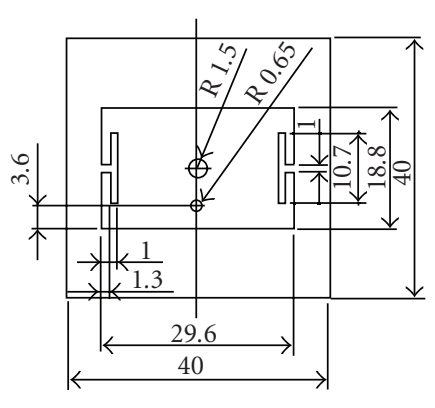

(b)

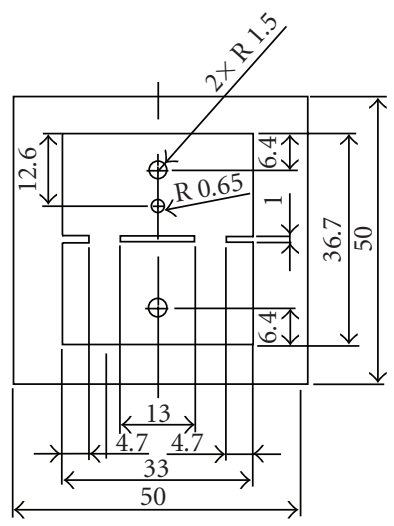

(d)
Figure 1: Geometry of (a) rectangular, (b) T-notched, (c) CoMPA, and (d) PCoMPA patches.

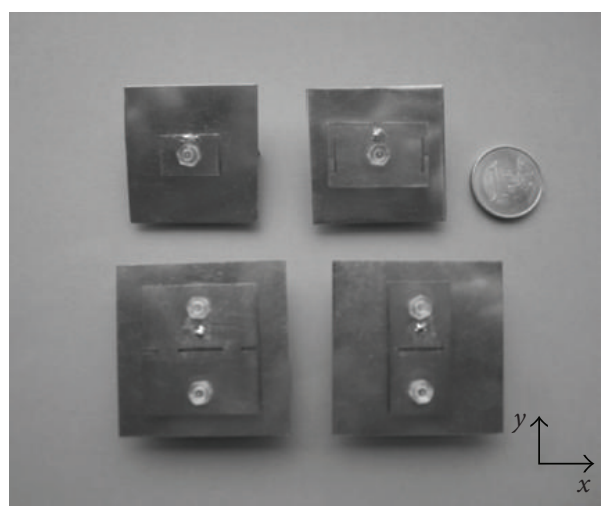

Figure 2: Photograph of realized prototypes.

\subsection{T-notched patch operating on $T M_{21}$ mode}

To enhance the gain of a rectangular patch operating on higher-order $\mathrm{TM}_{21}$ mode, the following patch topology modification has been done. A pair of T-shaped notches in so-called nonradiating edges has been introduced to modify vector surface current density. Each of the four corner stubs, formed by introducing the pair of T-notches, forces the original $x$-oriented current density $\mathbf{J}_{x}$ component of the $\mathrm{TM}_{21}$ mode to turn into the $y$-direction (into the stubs). As a result,

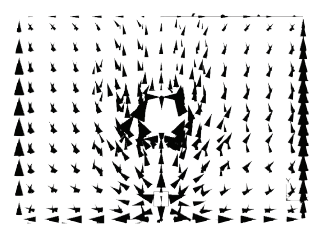

(a)

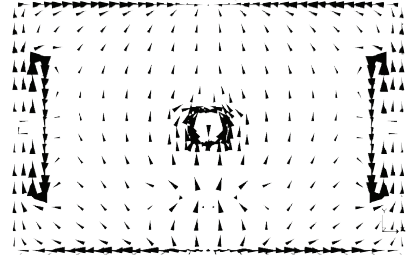

(b)
FIGURE 3: Vector surface current density of (a) reference rectangular patch operating on $\mathrm{TM}_{01}$ mode and (b) T-notched rectangular patch operating on $\mathrm{TM}_{21}$ mode at $10 \mathrm{GHz}$.

$\mathbf{J}_{y}$ component is dominant (see Figure $3(\mathrm{~b})$ ) and the radiator exhibits broadside radiation.

As the resonant frequency of $\mathrm{TM}_{21}$ mode is higher than that of $\mathrm{TM}_{01}$ mode, outer patch dimensions have to be increased to maintain the same resonant frequency. That is why the effective area with in-phase source current density is extended with consequent enhancement of the gain.

\subsection{Slotted and notched patches operating on $\mathrm{TM}_{03}$ mode}

The second type of presented patch topology employs PEs in the shape of transverse slot placed in the middle of the patch. This structure operates on $\mathrm{TM}_{03}$ mode. The slot, that is approximately $\lambda_{g} / 2$ long with the width of a fraction of $\lambda_{g}$, causes the fact that second/even current wavelength flows around the slot. The currents in the vicinity of the slot circumference thus cancel their contribution to the radiated fields due to the out-phase orientation on opposite sides of the slots. Specific patch topology with collinear in-phase distribution with two source areas thus arises, see Figure 4(a), which lead us to the name "collinear microstrip patch antenna" (CoMPA). So far, collinear arrays have been implemented just in wire [8], coaxial [12], or microstrip transmission line structures $[13,14]$.

As the next step for the gain enhancement, this radiator has been laterally extended and a pair of lateral transverse notches, with the length of approximately $\lambda_{g} / 4$, has been added. The current distribution of $\mathrm{TM}_{03}$ mode remains the same and a phenomenon of current flow around the slot and notches is presented again, see Figure 4(b). As a result, $y$-oriented current density $\mathbf{J}_{y}$ component is dominant on the surface of the patch; and the radiator exhibits broadside linear polarized radiation with a further gain enhancement. Domination of the $\mathbf{J}_{y}$ component on most of the patch surface is actually a necessary condition for reasonable low cross-polar level. This patch topology is further referred to as "planar collinear microstrip patch antenna" (PCoMPA).

\subsection{Synthesis and modeling procedure}

A synthesis based on the above-mentioned current distribution of operating higher-order mode on the patch consists of two steps: initial patch design based on a selection of operational mode with placement of PEs into the mentioned 
TABLE 1: Simulated and measured gain.

\begin{tabular}{l|c|c|c|c}
\hline Antenna & Simulated directivity [ dBi] & Simulated efficiency [\%] & Simulated gain [ dBi] & Measured gain[ dBi] \\
\hline Reference rectangular patch & 10.2 & 91.3 & 9.8 & 11.7 \\
T-notched patch & 12.0 & 94.2 & 11.1 & 11.5 \\
TM $_{03}$ CoMPA & 11.2 & 97.7 & 13.2 & 10.4 \\
TM $_{03}$ PCoMPA & 13.5 & 93.8 & 12.3 \\
\hline
\end{tabular}

TABle 2: Measured bandwidth and beamwidth.

\begin{tabular}{l|c|c|c|c}
\hline Antenna & BW[MHz] & BW[\%] & $\theta_{3 \mathrm{~dB}}\left[^{\circ}\right]$ in E plane & $\theta_{3 \mathrm{~dB}}\left[^{\circ}\right]$ in $\mathrm{H} \mathrm{plane}$ \\
\hline Reference rectangular patch & 620 & 6.2 & 43 & 48 \\
T-notched patch & 810 & 8.1 & 40 & 41 \\
TM $_{03}$ CoMPA & 625 & 6.3 & 25 & 55.5 \\
TM $_{03}$ PCoMPA & 284 & 2.9 & 26 & 35 \\
\hline
\end{tabular}

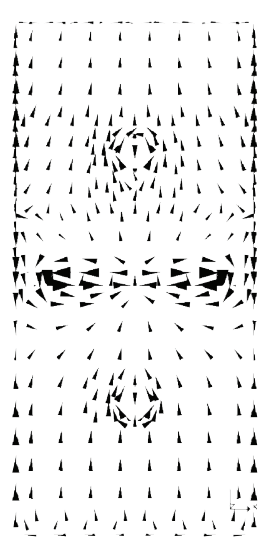

(a)

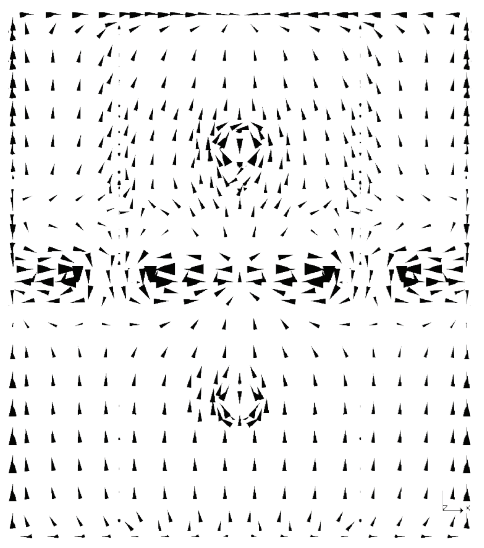

(b)
Figure 4: Vector surface current density of (a) CoMPA and (b) PCoMPA, operating on $\mathrm{TM}_{03}$ mode at $10 \mathrm{GHz}$.

positions and consequent optimization of the patch topology dimensions by electromagnetic simulator. Here we use a method of moment-based Zeland IE3D simulator with builtin optimization procedures with the criteria of impedance matching and maximization of the gain at the design frequency. Simulation models have been built with the segmentation of 20 cells per wavelength. The model of the patch with $0.3 \mathrm{~mm}$ thickness has been used. The probe feeding was modeled by a vertical-localized port. Simulation with finite dielectric volume has been used for modeling the influence of a plastic support post that carries the patch over the ground plane. Electrical properties of the plastic post which is $6 \mathrm{~mm}$ in diameter have not been exactly known but a value of $\varepsilon_{r}=2$ and $\tan \delta=0.005$ has been supposed.

\section{SIMULATED AND MEASURED RESULTS}

\subsection{Reflection coefficient}

The reflection coefficient of all radiators, which has been measured with reflectometer Anritsu SiteMaster S400A, can be seen in Figure 5. The reflection coefficient of a reference

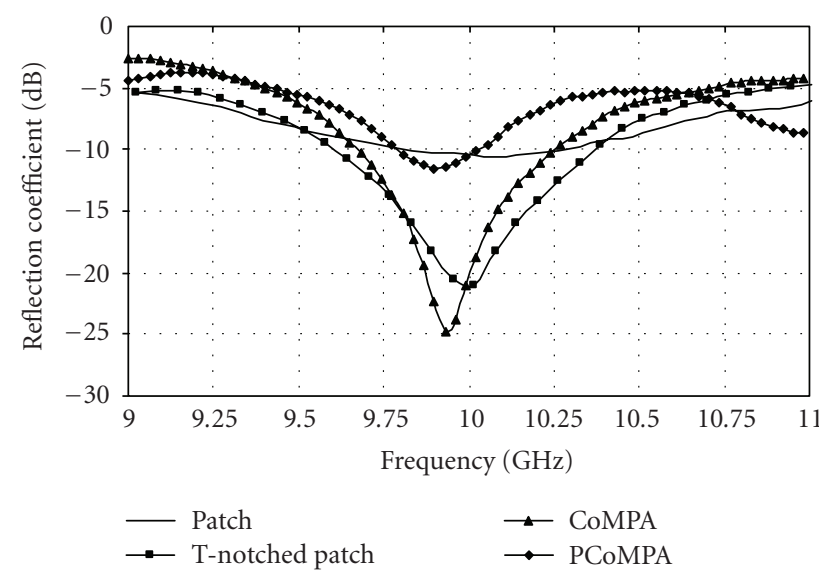

FIGURE 5: Measured reflection coefficient of reference rectangular, and gain enhanced T-notched, CoMPA, and PCoMPA patches.

rectangular patch is only about $-10 \mathrm{~dB}$ due to the noncompensated probe feed inductance.

\subsection{Radiation patterns}

The radiation patterns (see Figure 6) have been measured in a shielded anechoic chamber (the far field distance relation $d_{\text {far }}=2 D^{2} / \lambda_{0}$ has been achieved by setting the distance between AUTs at 4,55 m). All radiation patterns exhibit an increased level of cross polarization in the $\mathrm{H}$-plane. This is due to currents flowing on the vertical feed probe because a relatively large height of patches over the ground plane has been used. However, when comparing the simple rectangular and the T-notched patch in H-plane, we can see that the latter exhibits lower level of cross-polarization, see Figures 6(a) and $6(\mathrm{~b})$. This is due to the fact that the contributions of the currents, flowing vertically on the feed probe and horizontally on the patch in the direction of $x$-axis, are cancelled. A beam tilt of about $15^{\circ}$ can be seen in the E-plane of the reference patch, see Figure 6(a). The probe with vertical currents is placed closer to one of the edges of the patch. 

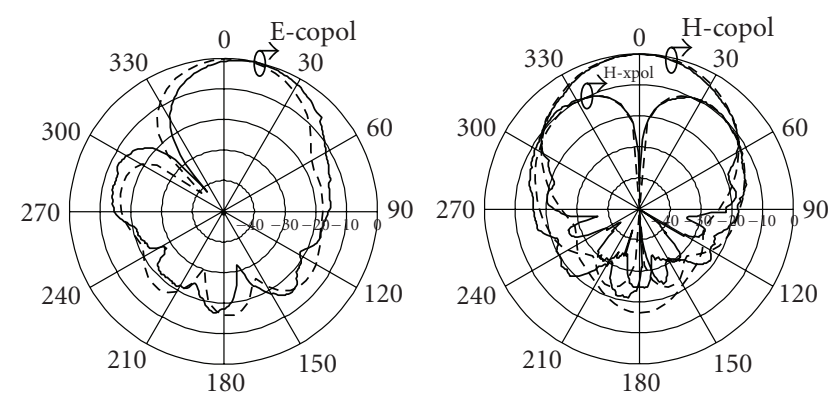

(a)

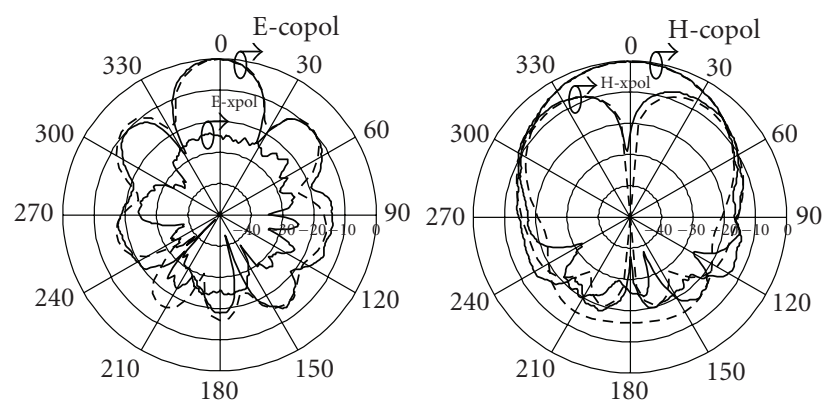

(c)
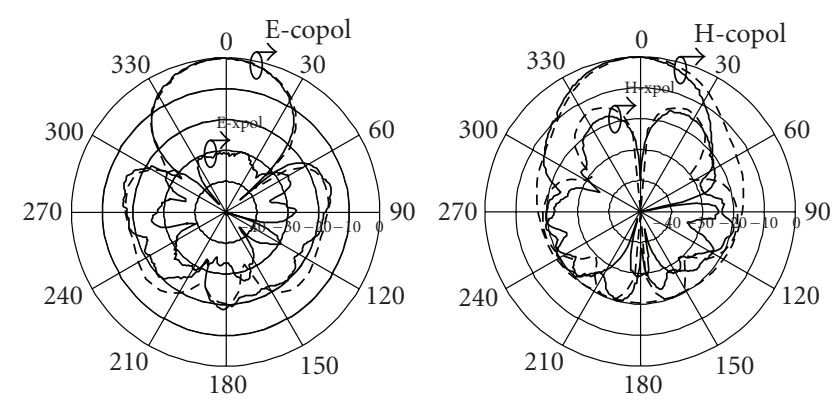

(b)
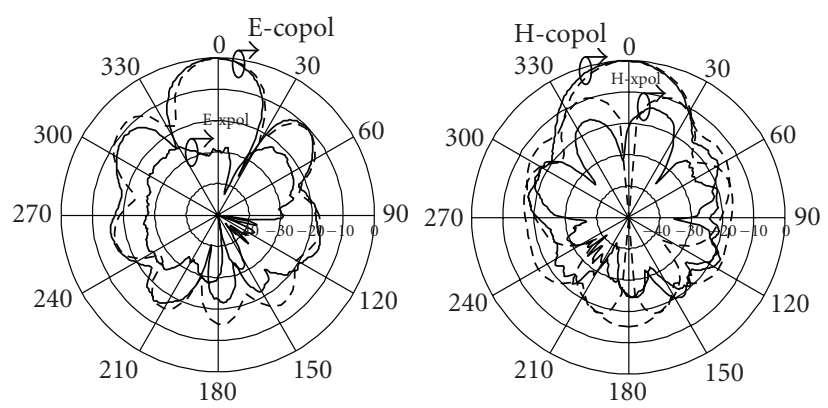

(d)

FIGURE 6: Measured radiation patterns of $10 \mathrm{GHz}$ patches in E-plane (left) and H-plane (right) of (a) reference rectangular patch, (b) Tnotched patch, (c) CoMPA, and (d) PCoMPA. Solid line: measurement; dash line: simulation.

\subsection{Gain}

The gain has been measured via the substitution method in the antenna anechoic chamber with the help of a doubleridged horn antenna (DRH $20[15])$. The details of the gain measurement settings can be found in [7]. It has been found that the measured gain of T-notched $\mathrm{TM}_{21}$ radiators, operating at $10 \mathrm{GHz}$, is $11.5 \mathrm{dBi}$ compared to $10.2 \mathrm{dBi}$ of a reference patch antenna. The measured gain of $\mathrm{TM}_{03}$ CoMPA and $\mathrm{TM}_{03}$ PCoMPA is $10.4 \mathrm{dBi}$ and $12.3 \mathrm{dBi}$, respectively (see Table 1). Comparison of measured $3 \mathrm{~dB}$ beamwidth confirms gain enhancement of modified patches compared to the reference rectangular one (see Table 2).

\section{CONCLUSION}

A novel technique for the gain enhancement of one-pointfed microstrip patches is presented. The principle is based on an extension of the source area. The dominant in-phase orientation of the surface current density is reached by using higher-order mode together with suitable patch topology modification. The modification is achieved by perturbation elements in the form of slots and notches, which force surface currents to flow dominantly in the direction of operating polarization and eliminating radiation from out-phase current areas. Two principal patch modifications, $\mathrm{TM}_{21}$ mode T-notched and $\mathrm{TM}_{03}$ mode CoMPA and PCOMPA patches, have been presented. A comparison of simulated and measured gain has been made; and a measured gain enhancement of $2.1 \mathrm{~dB}$ compared to reference rectangular patch gain has been reached in case of PCoMPA.
The advantages of proposed gain-enhanced patch topologies are the absence of any feed network, a simple construction, and low production costs. The drawback is the impossibility of controlling the amplitude and phase of inphase source current areas. Due to the resonant nature of operational mode, one can not efficiently control the sidelobe level mainly at CoMPA and PCoMPA patches.

\section{ACKNOWLEDGMENTS}

This work has been conducted at the Department of Electromagnetic Field of the Czech Technical University in Prague and supported by the grant of the Grant Agency of the Czech Republic no. 102/04/P131 "Multiband Planar Antennas with Compact-Shaped Radiators" and by the Czech Ministry of Education, Youth and Sports in the frame of the project Research in the Area of the Prospective Information and Navigation Technologies MSM 6840770014.

\section{REFERENCES}

[1] J. R. James and P. S. Hall, Eds., Handbook of Microstrip Antennas, Peter Peregrinus, London, UK, 1989.

[2] D. R. Jackson and N. G. Alexopoulos, "Gain enhancement methods for printed circuit antennas," IEEE Transactions on Antennas and Propagation, vol. 33, no. 9, pp. 976-987, 1985.

[3] X.-H. Shen, G. A. E. Vandenbosch, and A. R. Van de Capelle, "Study of gain enhancement method for microstrip antennas using moment method," IEEE Transactions on Antennas and Propagation, vol. 43, no. 3, pp. 227-231, 1995.

[4] R. Q. Lee and K. F. Lee, "Gain enhancement of microstrip antennas with overlaying parasitic directors," Electronics Letters, 
vol. 24 , no. 11, pp. 656-658, 1988.

[5] J. Romeu, C. Borja, and S. Blanch, "High directivity modes in the Koch island fractal patch antenna," in IEEE Antennas and Propagation Society International Symposium, vol. 3, pp. 16961699, Salt Lake, Utah, USA, July 2000.

[6] P. Hazdra, "Fractal antennas," diploma thesis, Czech Technical University in Prague, Prague, Czech Republic, 2003.

[7] M. Polivka and P. Hudec, "10 GHz broadside radiated linearly polarized patch witch enhanced gain," in CD-ROM Proceedings of the 35th European Microwave Conference, Paris, France, October 2005.

[8] C. S. Franklin, Brit. Patent 242342-1924, 1924.

[9] M. Polívka, A. Holub, P. Hudec, and M. Mazánek, "Collinear microstrip patch antenna," Radioengineering, vol. 14, no. 4, pp. 40-42, 2005.

[10] M. Polívka and A. Holub, "Planar version of collinear microstrip patch antenna," in Proceedings of the 16th International Conference on Microwaves, Radar, and Wireless Communications (MIKON '06), vol. 2, pp. 959-962, Krakow, Poland, May 2006.

[11] M. Polívka and A. Holub, CZ patent No. 296985, IPC H 01 Q 13/08, 2006.

[12] T. J. Judasz and B. B. Balsley, "Improved theoretical and experimental models for the coaxial colinear antenna," IEEE Transactions on Antennas and Propagation, vol. 37, no. 3, pp. 289296, 1989.

[13] K. Solbach, "Microstrip-Franklin antenna," IEEE Transactions on Antennas and Propagation, vol. 30, no. 4, pp. 773-775, 1982.

[14] R. Bancroft and B. Bateman, "An omnidirectional planar microstrip antenna," IEEE Transactions on Antennas and Propagation, vol. 52, no. 11, pp. 3151-3153, 2004.

[15] http://www.rfspin.com/, 24.6.2007.

\section{AUTHOR CONTACT INFORMATION}

Milan Polivka: Department of Electromagnetic Field,

Czech Technical University in Prague, Technická 2, 16627 Prague, Czech Republic; polivka@fel.cvut.cz

Alois Holub: Department of Electromagnetic Field, Czech Technical University in Prague, Technická 2, 16627 Prague, Czech Republic; alois.holub@seznam.cz 

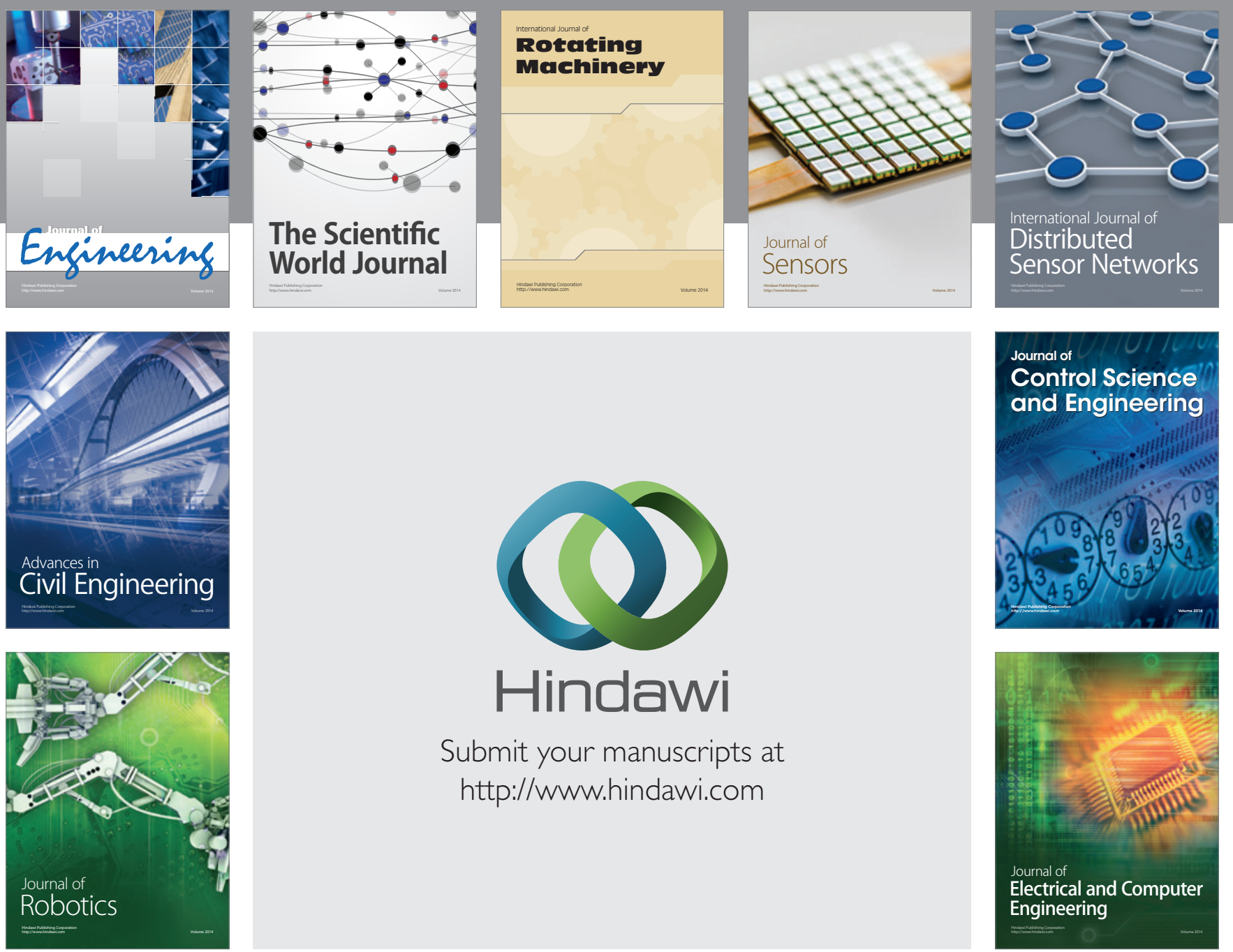

Submit your manuscripts at

http://www.hindawi.com
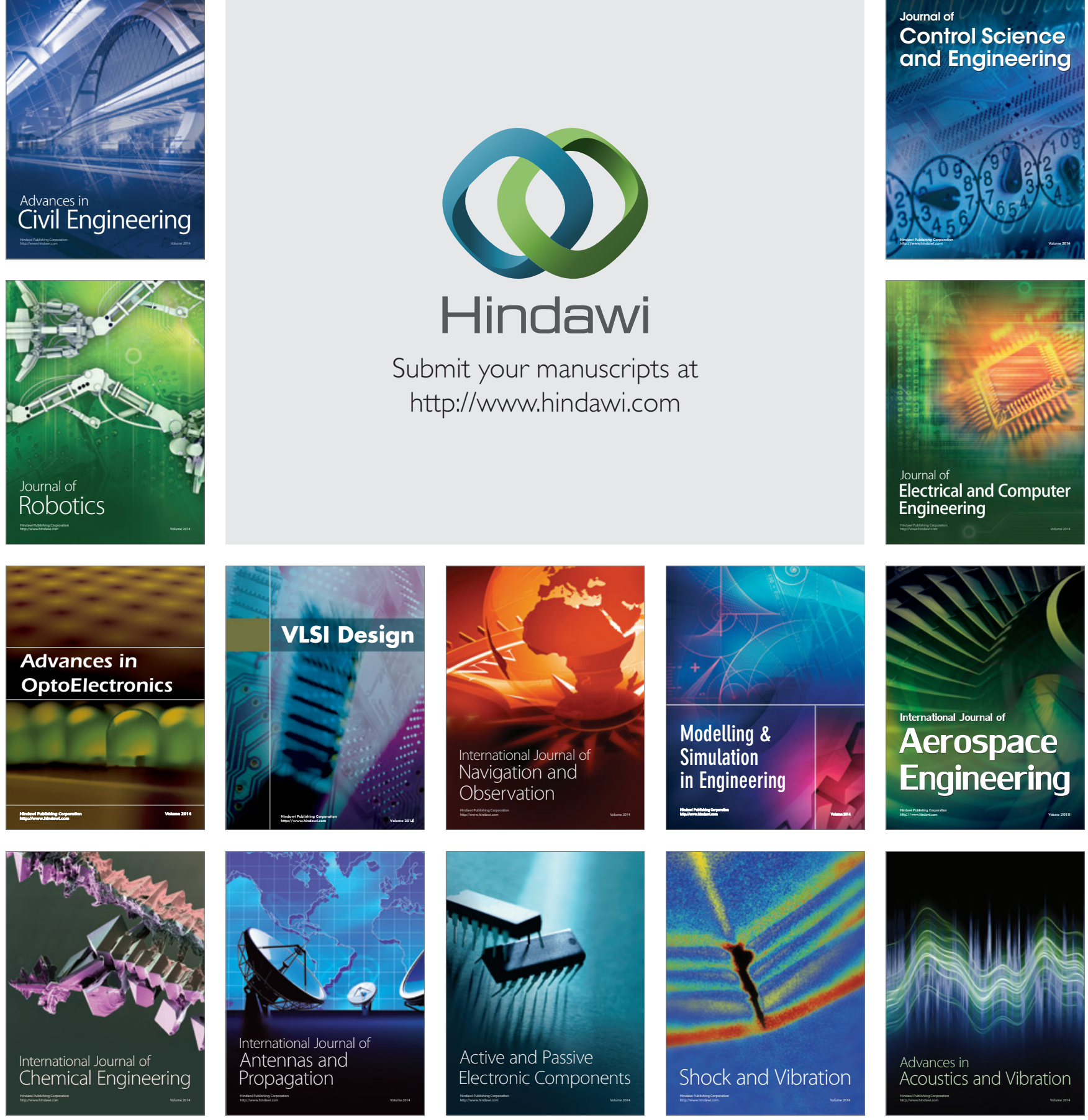